\title{
A Reduced-Order Fault Detection Filtering Approach for Continuous-Time Markovian Jump Systems with Polytopic Uncertainties
}

\author{
Lihong Rong, ${ }^{1,2}$ Xiuyan Peng, ${ }^{1}$ and Biao Zhang' \\ ${ }^{1}$ College of Automation, Harbin Engineering University, Harbin 150001, China \\ ${ }^{2}$ College of Information Technology, Heilongjiang Bayi Agricultural University, Daqing 163319, China \\ Correspondence should be addressed to Xiuyan Peng; pxygll@sina.com
}

Received 22 June 2016; Revised 18 September 2016; Accepted 27 October 2016; Published 11 January 2017

Academic Editor: Roberto Natella

Copyright ( 92017 Lihong Rong et al. This is an open access article distributed under the Creative Commons Attribution License, which permits unrestricted use, distribution, and reproduction in any medium, provided the original work is properly cited.

The fault detection (FD) reduced-order filtering problem is investigated for a family of continuous-time Markovian jump linear systems (MJLSs) with polytopic uncertain transition rates, which also include the totally known and partly unknown transition rates. Then, in accordance with the convexification techniques, a novel sufficient condition for the existence of FD reduced-order filter over MJLSs with deficient transition information is obtained in terms of linear matrix inequality (LMI), which can ensure the error augmented system with the FD reduced-order filter is randomly stable. In addition, a performance index is given to enhance the robustness of the residual system against deficient transition information and external disturbance, such that the error between the fault and the residual is made as small as possible to reinforce the faults sensitivity. Finally, the effectiveness of the proposed method is substantiated with two illustrative examples.

\section{Introduction}

Over the past few years, Markov jump linear systems (MJLSs) have been attracting extensive research attention in many engineering fields, such as energy system, solar thermal power generation system, networked control system, manufacturing system, and financial market system $[1,2]$. Many important results have been reported, such as a number of studies on the Markovian jump system on the filter design [3-5], state feedback controller design [6], output feedback controller design [7-12], stability analysis, and synthesis [1317]. In fact, MJLSs are very suited to dynamical model systems whose property is subject to random sudden variant due to abrupt external disturbance, shifting of the action spots of a nonlinear system, and repairs of components; thus, in order to ensure that the nonlinear system is randomly exponentially stable, the authors in [11] proposed a Markovian Lyapunov functional which was successfully used in the nonlinear systems. In essence, the transition rates (TRs) in the MJLSs are very important. A large number of traditional analyses and design results have been reported on condition that the TRs in the MJLSs are exactly known. However, it should be pointed out that all the mode transition rates cannot be acquired totally in lots of engineering plants; for example, the authors in [5] addressed two types of transition rates for the fault detection problem on discrete-time MJLSs. But, in fact, for the majority of MJLSs, there are three types of transition cases for the MJLSs, which are known, unknown, and polytopic uncertain TRs. For example, the authors in [2] proposed a control approach for continuous-time Markovian jump systems with time-varying delay and deficient transition descriptions. The authors in [3] presented a filtering method for two-dimensional continuoustime Markovian jump systems with partially accessible mode information. On the other hand, in many published papers, the unknown TRs and polytopic uncertain TRs in MJLSs have been taken into account separately. In reality, in a lot of actual conditions, there are the uncertain TRs and unknown TRs in MJLSs synchronously. To mention a few, the authors in [4] investigated a new approach to delay-dependent $H_{\mathrm{\infty}}$ filtering for discrete-time Markovian jump systems with the exactly known, partially unknown, and uncertain TRs concurrently, which was more rational and general to research on the 
comprehensive analysis of MJLSs. But there are few research results about fault detection of Markovian jump systems with the exactly known, partially unknown, and uncertain TRs concurrently, and the loss of sensor or actuator information can be efficiently modeled by means of Markov chain frameworks. This is the need to solve the main problem, which is one of the motivations for our research.

On another research frontier, the fault detection isolation and fault-tolerant control techniques have gotten a great amount of attention in the academic research and practical application because of the increasing demand for improving the system reliability and the safety of fail-safe control systems such as aerospace and nuclear plants (see [18-25]). The plant operation should be monitored in real time. When the components or instrument fault is found, the stable closed loop performance of the system has always be maintained, through the fault-tolerant control approach to realize the acceptable robust stability conditions of the system. Therefore, we should first study the fault detection methods. The basic design idea of FD is to use the effective methods to generate a residual signal and to determine a common diagnostic residual evaluation function and the threshold; then an alarm of fault is generated when the value of system residual is larger than the threshold [26]. Hence, in the process of fault detection, residual generation is a very important step; based on this, there are many basic approaches provided to generate robust residuals that are sensitive to faults, while being insensitive to unknown input and noise. There are many faults detection methods, such as full-state observer-based methods [27, 28], optimization-based approach [29], parity relations approach [30], unknown input observers [31], system identification methods [32], nonlinear approach [33, 34], artificial intelligence techniques $[35,36]$, and discrete event systems and hybrid systems [37-39]. In the above existing ways, the fault detection filter method is the most favoured method. However, in some complex engineering applications, highorder models are inevitably used to describe physical systems. This brings many difficulties in design of the corresponding FD filter in order to quickly detect faults. Moreover, to the knowledge of the authors, there are few results for the highefficiency FD reduced-order filter design. This motivates us to study this problem in order to reduce the complexity and FD rate of false positives and computation time of the FD filter design process and save storage space, so as to enhance the efficiency of the FD, which has great potential in practical applications.

In this paper, the chief aim is to design the FD reducedorder filter for a family of continuous-time MJLSs with uncertain transition probabilities, which is more general. By satisfying some performance indexes, the susceptibility to malfunction and the robustness against interference are both enhanced on residual outputs. Through the constructing of the residual generator, the FD reduced-order filter design scheme is converted to an $H_{\infty}$ reduced-order filtering design problem in order that the error between residual and malfunction is minimized at the $H_{\infty}$ level. Then, the sufficient condition for the existence of the FD reduced-order filter for the represented systems is obtained through linear matrix inequalities. In fact, compared with the fault detection reduced-order filter design for discrete-time Markov jump system with deficient transition information [25], the problem of fault detection for continuous-time MJLSs with deficient TRs should meet many requirements of detection performance and Markov jump process, which leads to the increase the difficulty of filter design. Therefore, to the best of our knowledge, the research on the fault detection reducedorder filters for continuous-time Markov jump system with deficient transition information is relatively few, which is the third motivation for this research.

Inspired by the aforementioned statements, in this paper, a reduced-order fault detection filtering approach for continuous-time MJLSs with polytopic uncertainties is firstly proposed. Then, the reduced-order $\mathrm{H}_{\infty}$ filter design problem is investigated by applying a linearisation approach, which casts the filter design into a convex optimisation problem. Finally, two examples are given to illustrate the effectiveness of the proposed design method. Compared with the existing result on FD filtering for the discrete-time Markovian jump linear systems, the main contributions of the work in this paper are twofold: (i) The filtering problem for a class of continuous-time MJLSs with defective transition information, which simultaneously includes the known, partially unknown, and polytopic-type uncertain TPs, is considered. The corresponding filter design results are expected to be more general and thereby more practicable. (ii) This proposed design approach has been applied to a vertical take-off and landing helicopter system, which can improve the sensitivity of fault detection and reduce the fault detection rate of false positives.

This paper is organized as follows. Section 2 formulates the mathematical model of the system; then, many preliminary results are shown. The sufficient condition of FD filter for the underlying system is established in Section 3. Section 4 describes two simulation cases study and results to point out the effectiveness of the proposed approach. Section 5 presents the conclusion of the this paper.

Notations. Throughout this paper, for real symmetric matrix $P, P>0$ means that $P$ is positive definite and $*$ represents the symmetric element. $\mathbb{R}^{n}$ denotes the $n$-dimensional Euclidean space, $\mathbb{R}^{m \times n}$ denotes the set of all $m \times n$ real matrices, and $N$ represents a positive integer. $\|\cdot\|$ denotes the Euclidean norm for vectors, $l_{2}[0, \infty)$ represents the space of square integrable vector functions over $\omega=\{\omega(t)\} \in l_{2}[0, \infty)$, and its norm is given by $\|\omega\|_{2}=\sqrt{\int_{0}^{\infty}\|\omega\|^{2} d t}$. $E[\cdot]$ stands for the mathematical expectation.

\section{Problem Formulation}

In this section, we will consider a continuous-time MJLS on a complete rate space of the form:

$$
\begin{aligned}
\dot{x}(t)= & A(\varsigma(t)) x(t)+B(\varsigma(t)) u(t)+E(\varsigma(t)) \omega(t) \\
& +F(\varsigma(t)) f(t), \\
y(t)= & C(\varsigma(t)) x(t)+D(\varsigma(t)) \omega(t)+H(\varsigma(t)) f(t),
\end{aligned}
$$


where $u(t) \in \mathbb{R}^{n_{u}}$ is the known control input, $y(t) \in \mathbb{R}^{n_{y}}$ is the controlled output, $x(t) \in \mathbb{R}^{n_{x}}$ represents the plant state, $f(t) \in \mathbb{R}^{n_{f}}$ is the fault signal to be detected, $\omega(t) \in \mathbb{R}^{n_{d}}$ is the exogenous disturbance signal, and $u(t), \omega(t)$, and $f(t)$ are assumed to belong to $l_{2}[0, \infty)$. $\{\varsigma(t), t \geq 0\}$ is a continuoustime homogeneous Markov chain, which takes values in a finite set $S=\{1,2, \ldots, n\}$ and mode transition rates (TRs) are defined as

$$
\begin{aligned}
\text { Prob }\{\varsigma(t+h)=j \mid \varsigma(t)=g\} \\
\quad= \begin{cases}\lambda_{g j} h+o(h), & \varsigma(t) \text { jumps from } g \text { to } j, \\
1+\lambda_{g g} h+o(h), & \text { otherwise, }\end{cases}
\end{aligned}
$$

where $h>0, \lim _{h \rightarrow 0}(o(h) / h)=0$, for all $g \neq j, \lambda_{g j} \geq 0$, and $\lambda_{g g}=-\sum_{j=1, j \neq g}^{N} \lambda_{g j}$. For $\varsigma(t)=g, g \in S$, the system matrices of the $g$ th mode are denoted by $(\mathrm{Ag}, \mathrm{Bg}, \mathrm{Cg}, \mathrm{Dg})$, which are known real matrices. In this paper, system (1) is assumed randomly stable, which is a precondition for model design.

Moreover, the TRs of the Markov process are regarded as polytopic uncertain and partly available; in other words, the transition rate matrix (TRM) $\Lambda=\left\{\lambda_{g j}\right\}$ is deemed to belong to a known polytope $P_{\Lambda}$ with vertices $\Lambda_{s}$.

$$
P_{\Lambda}:=\left\{\Lambda \mid \Lambda=\sum_{s=1}^{M} \alpha_{s} \Lambda_{s} ; \alpha_{s} \geq 0, \sum_{s=1}^{M} \alpha_{s}=1\right\},
$$

where vertices $\Lambda_{s}=\left[\lambda_{g j}\right]_{N \times N}, g, j \in S, S=1,2, \ldots, M$, are still given in TRM containing unknown and uncertain factors. For example, for system (1) with four variation modes, the TRM is expressed as

$$
\left[\begin{array}{llll}
\lambda_{11} & \hat{\lambda}_{12} & \tilde{\lambda}_{13} & \lambda_{14} \\
\tilde{\lambda}_{21} & \lambda_{22} & \hat{\lambda}_{23} & \lambda_{24} \\
\lambda_{31} & \lambda_{32} & \tilde{\lambda}_{33} & \hat{\lambda}_{34} \\
\tilde{\lambda}_{41} & \lambda_{42} & \lambda_{43} & \tilde{\lambda}_{44}
\end{array}\right],
$$

where the polytopic uncertainties and unknown TRs are represented as the superscripts labeled with " " and "—," separately, and the others are known TRs. In order to make the notational more clearly, for all $g \in S$, we denote $S=$ $S_{k}^{(g)} \cup S_{u c}^{(g)} \cup S_{u k}^{(g)}$ as follows:

$$
\begin{aligned}
& S_{k}^{(g)}:=\left\{j: \lambda_{g j} \text { is known }\right\}, \\
& S_{u c}^{(g)}:=\left\{j: \widetilde{\lambda}_{g j} \text { is uncertain }\right\}, \\
& S_{u k}^{(g)}:=\left\{j: \hat{\lambda}_{g j} \text { is unknown }\right\} .
\end{aligned}
$$

Also, we define $\lambda_{u k}^{(g v)}:=\sum_{j \in S_{u k}^{(g)}} \hat{\lambda}_{g j}=1-\sum_{j \in S_{k}^{(g)}} \lambda_{g j}-$ $\sum_{j \in S_{u c}^{(g)}} \tilde{\lambda}_{g j}^{(g v)}$.

Remark 1. The transition rates of the MJLSs $\{\varsigma(t), t \geq 0\}$ have been universally assumed to be some known, some unknown, and some uncertain within given intervals. Hence, the TRM considered in this article is more natural to the MJLSs, which includes the previous three cases. Then, we are interested in designing an FD filter for the underlying system, and its desired structure is considered to be

$$
\begin{aligned}
\dot{x}_{F}(t) & =A_{F}(\varsigma(t)) x_{F}(t)+B_{F}(\varsigma(t)) y(t), \\
r_{F}(t) & =C_{F}(\varsigma(t)) x_{F}(t)+D_{F}(\varsigma(t)) y(t),
\end{aligned}
$$

where $x_{F}(t) \in \mathbb{R}^{n}$ is the state estimation of filter, $r_{F}(t) \in$ $\mathbb{R}^{f}$ is the residual, and $A_{F}(\varsigma(t)), B_{F}(\varsigma(t)), C_{F}(\varsigma(t)), D_{F}(\varsigma(t))$, $\forall \varsigma(t) \in I$ are the matrices to be calculated.

Define $\widetilde{x}(t):=\left[\begin{array}{ll}x^{T}(t) & x_{F}{ }^{T}(t)\end{array}\right]^{T}, e(t):=r_{F}(t)-f(t)$. Then, by augmenting (1) and (6), the error augmented system is obtained as follows:

$$
\begin{aligned}
& \dot{\tilde{x}}(t)=\widetilde{A}(\varsigma(t)) \widetilde{x}(t)+\widetilde{B}(\varsigma(t)) \psi(t), \\
& e(t)=\widetilde{C}(\varsigma(t)) \widetilde{x}(t)+\widetilde{D}(\varsigma(t)) \psi(t),
\end{aligned}
$$

where $\psi(t)=\left[\begin{array}{lll}u^{T}(t) & \omega^{T}(t) & f^{T}(t)\end{array}\right]^{T}$ and

$$
\begin{aligned}
& \widetilde{A}(\varsigma(t))=\left[\begin{array}{cc}
A(\varsigma(t)) & 0 \\
B_{F}(\varsigma(t)) C(\varsigma(t)) & A_{F}(\varsigma(t))
\end{array}\right], \\
& \widetilde{B}(\varsigma(t)) \\
& =\left[\begin{array}{ccc}
B(\varsigma(t)) & E(\varsigma(t)) & F(\varsigma(t)) \\
0 & B_{F}(\varsigma(t)) D(\varsigma(t)) & B_{F}(\varsigma(t)) H(\varsigma(t))
\end{array}\right], \\
& \widetilde{C}(\varsigma(t))=\left[\begin{array}{ll}
D_{F}(\varsigma(t)) C(\varsigma(t)) & C_{F}(\varsigma(t))
\end{array}\right], \\
& \widetilde{D}(\varsigma(t)) \\
& =\left[\begin{array}{lll}
0 & D_{F}(\varsigma(t)) D(\varsigma(t)) & D_{F}(\varsigma(t)) H(\varsigma(t))-I
\end{array}\right] .
\end{aligned}
$$

In fact, the error augmented system (7) is also an MJLS with deficient TRM in (4). We recommend the definitions of stochastic stability of the Markovian jump system for system (7), which are necessary for the next step of progress.

Definition 2 (see [2]). A continuous-time stochastic system (7) is said to be randomly stable if, for $\omega(t)=0, t \geq 0$, and every initial condition $\widetilde{x}(0) \in \mathbb{R}^{n_{x}}$ and $r(0) \in S$. Then, the following holds: $E\left\{\int_{0}^{\infty}\|\widetilde{x}(h), \widetilde{x}(0), r(0)\|^{2} d h\right\}<\infty$.

Definition 3 (see [6]). Given the disturbance input $\omega(t) \epsilon$ $l_{2}[0, \infty)$ and a scalar $\varsigma>0$, system (7) is randomly stable and has an $H_{\infty}$ performance index $\varsigma$ if the following two conditions are satisfied:

(1) When $\omega(t)=0, t \geq 0$, system (7) is randomly stable in the sense of Definition 2.

(2) When $\omega(t) \neq 0, t \geq 0$, under zero initial conditions, the following inequality holds:

$$
E\left\{\int_{0}^{\infty} e^{T}(t) e(t) d t\right\}<\varsigma^{2} E\left\{\int_{0}^{\infty} \omega^{T}(t) \omega(t) d t\right\} .
$$


As a consequence, the main purposes of this paper are to determine matrices $\left\{A_{F}(\varsigma(t)), B_{F}(\varsigma(t)), C_{F}(\varsigma(t)), D_{F}(\varsigma(t))\right\}$ in system (6), such that the augmented error system (7) is randomly stable with a reliable $H_{\infty}$ performance level with deficient transition information. Finally, the continuous-time MJLS (1) will be assumed to be stable in the end. Moreover, in order to detect the fault $f(t)$, the residual evaluation function is designed as $J(r(L))=\sqrt{\int_{k_{0}}^{k_{0}+L} r^{T}(t) r(t) d t}$, where $k_{0}$ denotes the initial evaluation time instant. The fault $f(t)$ can be detected by the following steps.

(i) Select a threshold $J_{\text {th }} \triangleq \sup _{d \in l_{2}, f=0} E[J(r(L))]$.

(ii) Based on the above result, the fault $f_{k}$ can be detected by comparing $J(r(L))$ and $J_{\text {th }}$.

(iii) When $J(r(L)) \geq J_{\text {th }}$, there are some faults; we should give an alarm; when $J(r(L))<J_{\text {th }}$, there are no faults.

Before proceeding further, it is worth briefly reviewing the following useful lemma on the error augmented system (7) with completely known TRs, which is given for the derivation of the latter results.

Lemma 4 (see [10]). For the error augmented system (7) with totally known transition mode information and a given scalar $\xi>0$, the coupled inequalities

$$
\left[\begin{array}{cc}
\widetilde{A}_{g}^{T} Q_{g}+Q_{g} \widetilde{A}_{g}+\eta_{g}+\widetilde{C}_{g}^{T} \widetilde{C}_{g} & Q_{g} \widetilde{B}_{g}+\widetilde{C}_{g}^{T} \widetilde{D}_{g} \\
* & -\left(\xi^{2} I-\widetilde{D}_{g}^{T} \widetilde{D}_{g}\right)
\end{array}\right]
$$

$<0, \quad \forall g \in S$, where $\eta_{g}:=\sum_{j=1}^{N} \lambda_{g j} Q_{j}$ have resolvable matrices $Q=$ $\left\{Q_{1}, Q_{2}, \ldots, Q_{N}\right\}$ such that MJLS (7) with totally known TRs is randomly stable with an $H_{\infty}$ performance index $\xi$.

\section{Main Results}

In the above section, firstly, we introduce an $H_{\infty}$ performance analysis criterion for the error augmented system (7) and further focus on the design of the FD reduced-order filter for MJLS (1) with deficient mode information.

3.1. $H_{\infty}$ FD Reduced-Order Filter Performance Analysis. The following lemma presents an $H_{\infty}$ FD reduced-order filter performance analysis result for the underlying augmented error system in (7) with deficient TRs.

Lemma 5. Let $\xi>0$ be a given scalar; if there are positivedefinite symmetric matrices $Q=\left\{Q_{1}, Q_{2}, \ldots, Q_{N}\right\}$ such that LMI (11) holds, then the error augmented system in (7) with incomplete mode transition information is randomly stable with a guaranteed $H_{\infty}$ performance index $\xi$ and satisfies (9).

$$
\begin{aligned}
\Psi_{g j}^{(v)}=\left[\begin{array}{ccc}
-I & \widetilde{C}_{g} & \widetilde{D}_{g} \\
* & \widetilde{A}_{g}^{T} Q_{g}+Q_{g} \widetilde{A}_{g}+\widetilde{\eta}_{g j}^{(v)} & Q_{g} \widetilde{B}_{g} \\
* & * & -\xi^{2} I
\end{array}\right]<0, \\
\quad j \in S_{u k}^{(g)}, v=1,2, \ldots, M,
\end{aligned}
$$

where

$$
\begin{aligned}
& \tilde{\eta}_{g j}^{(v)}= \begin{cases}\eta_{k}^{(g)}+\eta_{u c}^{(g v)}-\left(\lambda_{k}^{(g)}+\lambda_{u c}^{(g v)}\right) \eta_{j}, & j \in S_{u k}^{(g)}, \text { if } g \in S_{k}^{(g)} \cup S_{u c}^{(g)}, \\
\eta_{k}^{(g)}+\eta_{u c}^{(g v)}+\lambda_{l}^{(g)} \eta_{g}-\left(\lambda_{l}^{(g)}+\lambda_{k}^{(g)}+\lambda_{u c}^{(g v)}\right) \eta_{j}, \quad j \in S_{u k}^{(g)}, \text { if } g \in S_{u k}^{(g)},\end{cases} \\
& \eta_{k}^{(g)}=\sum_{j \in S_{k}^{(g)}} \lambda_{g j} Q_{j}, \\
& \eta_{u c}^{(g v)}=\sum_{j \in S_{u c}^{(g)}} \tilde{\lambda}_{g j}^{(v)} Q_{j}, \\
& \lambda_{k}^{(g)}=\sum_{j \in S_{k}^{(g)}} \lambda_{g j}, \\
& \lambda_{u c}^{(g v)}=\sum_{j \in S_{u c}^{(g)}} \tilde{\lambda}_{g j}^{(v)} .
\end{aligned}
$$

Proof. By virtue of Lemma 4, it is shown that system (7) with totally known transition probabilities is randomly stable with an $H_{\infty}$ performance $\xi$, when matrix inequality (10) holds. Now because the diagonal elements in the transition probabilities matrix may not be known, the proof of Lemma 5 should be divided into two cases to analyze; that is, $g \in$ $S_{k}^{(g)} \cup S_{u c}^{(g)}$ (Case 1) and $g \in S_{u k}^{(g)}$ (Case 2), respectively.

Case $1\left(g \in S_{k}^{(g)} \cup S_{u c}^{(g)}\right)$. In this case, $g \in S_{k}^{(g)} \cup S_{u c}^{(g)}$ indicates that $\lambda_{g g}$ is known or uncertain; then it is equivalent to $\lambda_{k}^{(g)}+\lambda_{u c}^{(g s)} \leq 0$. 
First of all, we consider the case that $\lambda_{k}^{(g)}+\lambda_{u c}^{(g s)}<$ 0 . Noticing that, with incomplete probabilities information, $\sum_{j=1}^{N} \lambda_{g j} Q_{j}$ in (10) can be dealt with,

$$
\begin{aligned}
\sum_{j=1}^{N} \lambda_{g j} Q_{j}= & \sum_{j \in S_{k}^{(g)}} \lambda_{g j} Q_{j}+\sum_{j \in S_{u k}^{(g)}} \hat{\lambda}_{g j} Q_{j} \\
& +\sum_{j \in S_{u c}^{(g)}}\left(\sum_{v=1}^{M} \beta_{v} \tilde{\lambda}_{g j}^{(v)}\right) Q_{j} \\
= & \sum_{j \in S_{k}^{(g)}} \lambda_{g j} Q_{j} \\
& +\left(-\lambda_{k}^{(g)}-\lambda_{u c}^{(g v)}\right) \sum_{j \in S_{u k}^{(g)}} \frac{\hat{\lambda}_{g j}^{(g)}-\lambda_{u c}^{(g v)}}{-Q_{j}} \\
& +\sum_{v=1}^{M} \beta_{v} \sum_{j \in S_{u c}^{(g)}} \tilde{\lambda}_{g j}^{(v)} Q_{j} \\
= & \eta_{k}^{(g)}+\left(-\lambda_{k}^{(g)}-\lambda_{u c}^{(g v)}\right) \sum_{j \in S_{u k}^{(g)}} \frac{\hat{\lambda}_{g j}^{(g)}-\lambda_{u c}^{(g v)}}{-\lambda_{j}} \\
& +\sum_{v=1}^{M} \beta_{v} \eta_{u c}^{(g v)},
\end{aligned}
$$

where

$$
\begin{aligned}
& \eta_{k}^{(g)}=\sum_{j \in S_{k}^{(g)}} \lambda_{g j} Q_{j}, \\
& \eta_{u c}^{(g v)}=\sum_{j \in S_{u c}^{(g)}} \tilde{\lambda}_{g j}^{(v)} Q_{j}, \\
& \lambda_{k}^{(g)}=\sum_{j \in S_{k}^{(g)}} \lambda_{g j}, \\
& \lambda_{u c}^{(g v)}=\sum_{j \in S_{u c}^{(g)}} \tilde{\lambda}_{g j}^{(v)},
\end{aligned}
$$

and $\hat{\lambda}_{g j}\left(j \in S_{u k}^{(g)}\right)$ are unknown elements; and $\sum_{v=1}^{M} \beta_{v} \tilde{\lambda}_{g j}^{(v)}$, $\forall j \in S_{u c}^{(g)}$ represents the polytopic uncertain elements.

As $0 \leq \beta_{v} \leq 1, \sum_{v=1}^{M} \beta_{v}=1$, and $0 \leq \widehat{\lambda}_{g j} /\left(-\lambda_{k}^{(g)}-\lambda_{u c}^{(g v)}\right) \leq$

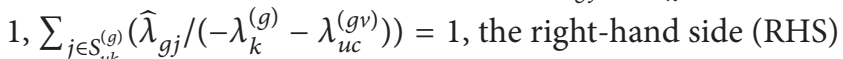
of inequality (13) is sorted out for the following expression:

$$
\begin{aligned}
& \operatorname{RHS}(13)=\sum_{v=1}^{M} \beta_{v} \sum_{j \in S_{u k}^{(g)}} \frac{\hat{\lambda}_{g j}}{-\lambda_{k}^{(g)}-\lambda_{u c}^{(g v)}}\left(\eta_{k}^{(g)}+\eta_{u c}^{(g v)}\right. \\
&\left.-\left(\lambda_{k}^{(g)}+\lambda_{u c}^{(g v)}\right) \eta_{j}\right) .
\end{aligned}
$$

Thus, for $0 \leq \beta_{v} \leq 1$ and $0 \leq \hat{\lambda}_{g j} \leq-\left(\lambda_{k}^{(g)}+\lambda_{u c}^{(g v)}\right)$, the lefthand side (LHS) of inequality (10) can be obtained as follows:

$$
\begin{aligned}
\operatorname{LHS}(10)=\sum_{v=1}^{M} \beta_{v} \sum_{j \in S_{u c}^{(g)}} \frac{\hat{\lambda}_{g j}}{-\lambda_{k}^{(g)}-\lambda_{u c}^{(g s)}} \Psi_{g j}^{(v)}, \\
\quad j \in S_{u k}^{(g)}, \quad v=1,2, \ldots, M,
\end{aligned}
$$

where

$$
\begin{aligned}
& \Psi_{g j}^{(v)}=\left[\begin{array}{ccc}
-I & \widetilde{C}_{g} & \widetilde{D}_{g} \\
* & \widetilde{A}_{i}^{T} Q_{g}+Q_{g} \widetilde{A}_{g}+\widetilde{\eta}_{g j}^{(v)} & Q_{g} \widetilde{B}_{i} \\
* & * & -\xi^{2} I
\end{array}\right], \\
& \widetilde{\eta}_{g j}^{(v)}:=\eta_{k}^{(g)}+\eta_{u c}^{(g v)}-\left(\lambda_{k}^{(g)}+\lambda_{u c}^{(g v)}\right) \eta_{j} .
\end{aligned}
$$

When $\Psi_{g j}^{(v)}<0$ in (16), inequality (10) holds.

Secondly, we consider the case that $\lambda_{k}^{(g)}+\lambda_{\mathcal{u c}}^{(g v)}=0$.

In fact, if $\lambda_{k}^{(g)}+\lambda_{u c}^{(g s)}=0$, then all the items in the $g$ th row are fully known. Inequality (11) is obtained through the linear transformation with $\widetilde{\eta}_{g j}^{(v)}:=\eta_{k}^{(g)}+\eta_{u c}^{(g s)}$.

In conclusion, when the unknown elements $\widehat{\lambda}_{g j}, g \neq j$, are not the diagonal elements $\hat{\lambda}_{g g}$, inequality (10) can be converted to (11).

Case $2\left(g \in S_{u k}^{(g)}\right)$. Firstly, it is the case that $\widehat{\lambda}_{g g}<-\left(\lambda_{k}^{(g)}+\lambda_{u c}^{(g v)}\right)$.

Identically, for this case the term $\sum_{j=1}^{N} \lambda_{g j} Q_{j}$ in (10) can be processed into

$$
\begin{aligned}
& \sum_{j=1}^{N} \lambda_{g j} Q_{j}=\sum_{j \in S_{k}^{(g)}} \lambda_{g j} Q_{j}+\hat{\lambda}_{g g} Q_{g}+\sum_{j \in S_{u k}^{(g)}, j \neq g} \hat{\lambda}_{g j} Q_{j} \\
& \quad+\sum_{j \in S_{u c}^{(g)}}\left(\sum_{v=1}^{M} \beta_{v} \tilde{\lambda}_{g j}^{(v)}\right) Q_{j}=\eta_{k}^{(g)}+\hat{\lambda}_{g g} Q_{g} \\
& \quad-\left(\hat{\lambda}_{g g}+\lambda_{k}^{(g)}+\lambda_{u c}^{(g v)}\right) \\
& \quad \sum_{j \in S_{u k}^{(g)}, j \neq g} \frac{\hat{\lambda}_{g j}}{-\hat{\lambda}_{g g}-\lambda_{k}^{(g)}-\lambda_{u c}^{(g v)}} Q_{j}+\sum_{v=1}^{M} \beta_{v} \eta_{u c}^{(g v)},
\end{aligned}
$$

where $\eta_{k}^{(g)}:=\sum_{j \in S_{k}^{(g)}} \lambda_{g j} Q_{j}$ and $\eta_{u c}^{(g v)}:=\sum_{j \in S_{u c}^{(g)}} \tilde{\lambda}_{g j}^{(v)} Q_{j}$.

Similarly, it follows from $0 \leq \beta_{v} \leq 1, \sum_{v=1}^{M} \beta_{v}=1$, and $0 \leq \hat{\lambda}_{g j} /\left(-\hat{\lambda}_{g g}-\lambda_{k}^{(g)}-\lambda_{u c}^{(g v)}\right) \leq 1, \sum_{j \in S_{u k}^{(g)}, j \neq g}\left(\hat{\lambda}_{g j} /\left(-\hat{\lambda}_{g g}-\right.\right.$ $\left.\left.\lambda_{k}^{(g)}-\lambda_{u c}^{(g v)}\right)\right)=1$, that

$$
\begin{aligned}
\sum_{j=1}^{N} \lambda_{g j} Q_{j} & =\sum_{v=1}^{M} \beta_{v} \sum_{j \in S_{u k}^{(g)}, j \neq g} \frac{\widehat{\lambda}_{g j}}{-\lambda_{k}^{(g)}-\lambda_{u c}^{(g s)}-\widehat{\lambda}_{g g}}\left[\eta_{k}^{(g)}\right. \\
+\eta_{u c}^{(g v)} & \left.+\widehat{\lambda}_{g g} Q_{g}-\left(\widehat{\lambda}_{g g}+\lambda_{k}^{(g)}+\lambda_{u c}^{(g v)}\right) Q_{j}\right] .
\end{aligned}
$$


Correspondingly, for this case, LHS (10) can be rewritten as

$$
\begin{aligned}
& \operatorname{LHS}(10)=\sum_{v=1}^{M} \beta_{v} \sum_{j \in S_{u k}^{(g)}, j \neq g} \frac{\hat{\lambda}_{g j}}{-\lambda_{k}^{(g)}-\lambda_{u c}^{(g s)}-\hat{\lambda}_{g g}} \Psi_{g j}^{(v)}, \\
& \quad j \in S_{u k}^{(g)}, j \neq g, \quad v=1,2, \ldots, M,
\end{aligned}
$$

where

$$
\begin{aligned}
& \Psi_{g j}^{(v)}:=\left[\begin{array}{ccc}
-I & \widetilde{C}_{g} & \widetilde{D}_{g} \\
* & \widetilde{A}_{g}^{T} Q_{g}+Q_{g} \widetilde{A}_{g}+\widetilde{\eta}_{g j}^{(v)} & Q_{g} \widetilde{B}_{g} \\
* & * & -\xi^{2} I
\end{array}\right], \\
& \tilde{\eta}_{g j}^{(v)}:=\eta_{k}^{(g)}+\eta_{u c}^{(g v)}+\widehat{\lambda}_{g g} Q_{g}-\left(\lambda_{k}^{(g)}+\lambda_{u c}^{(g v)}+\widehat{\lambda}_{g g}\right) Q_{j} .
\end{aligned}
$$

It follows from (20) that (10) is equivalent to

$$
\Psi_{g j}^{(v)}<0, \quad j \in S_{u k}^{(g)}, j \neq g .
$$

To facilitate the calculation, we introduce a lower bound $\lambda_{l}^{(g)}$ for the unknown element $\hat{\lambda}_{g g}$; that is,

$$
\lambda_{l}^{(g)} \leq \hat{\lambda}_{g g}<-\lambda_{k}^{(g)}-\lambda_{u c}^{(g v)}
$$

which indicates that $\hat{\lambda}_{g g}$ can take different value in $\left[\lambda_{l}^{(g)},-\lambda_{k}^{(g)}-\lambda_{u c}^{(g v)}+\varepsilon\right]$ for any small value $\varepsilon<0$. Then $\hat{\lambda}_{g g}$ can be further expressed as follows:

$$
\hat{\lambda}_{g g}=-\xi \lambda_{k}^{(g)}-\xi \lambda_{u c}^{(g v)}+\xi \varepsilon+(1-\xi) \lambda_{l}^{(g)}
$$

where $0 \leq \xi \leq 1$. As $\hat{\lambda}_{g g}$ in (24) depends on $\xi$ linearly, therefore (22) only needs to be satisfied for $\xi=0$ and $\xi=1$; that is, (22) holds if and only if the following inequalities in (25)-(26) simultaneously hold:

$$
\Psi_{g j}^{(v)}<0, \quad j \in S_{u k}^{(g)}, j \neq g,
$$

where $\Psi_{g j}^{(v)}$ is defined in (21) with $\widetilde{\eta}_{g j}^{(v)}=\eta_{k}^{(g)}+\eta_{u c}^{(g v)}-\left(\lambda_{k}^{(g)}+\right.$ $\left.\lambda_{u c}^{(g v)}\right) Q_{j}+\varepsilon\left(Q_{g}-Q_{j}\right)$, and

$$
\Psi_{g j}^{(v)}<0, \quad j \in S_{u k}^{(g)}, j \neq g
$$

where $\Psi_{g j}^{(v)}$ is defined in (21) with $\widetilde{\eta}_{g j}^{(v)}=\eta_{k}^{(g)}+\eta_{u c}^{(g v)}-\left(\lambda_{k}^{(g)}+\right.$ $\left.\lambda_{u c}^{(g v)}\right) Q_{g}+\lambda_{l}^{(g)}\left(Q_{g}-Q_{j}\right)$.

As $\varepsilon$ is small enough, (25) is established only if

$$
\Psi_{g j}^{(v)}<0, \quad j \in S_{u k}^{(g)}, j \neq g,
$$

where $\Psi_{g j}^{(v)}$ is defined in (21) with $\widetilde{\eta}_{g j}^{(v)}=\eta_{k}^{(g)}+\eta_{u c}^{(g v)}-\left(\lambda_{k}^{(g)}+\right.$ $\left.\lambda_{u c}^{(g v)}\right) Q_{g}$, which is implied by (26) when $j=g, j \in S_{u k}^{(g)}$.

From what has been discussed above, despite the presence of uncertain and unknown terms in the transition probabilities matrix, the error augmented system (7) is randomly stable with an $H_{\infty}$ performance $\xi$ if (11) holds. This completes the proof.

Remark 6. Lemma 5 presents an $H_{\infty}$ performance analysis standard for a family of MJLSs with deficient TRs. However, it is shown that there are coupling terms in the system matrices inequality (11), where structural constraint significantly augments the level of design conservatism. Thus, it incurs some difficulties for fault detection filter synthesis problem. To overcome these difficulties, the slack matrix method can be adopted here in order to obtain the following improved criterion for the error augmented system (7).

3.2. Design of $H_{\infty}$ Reduced-Order FD Filter. The next step is to translate the $H_{\infty}$ FD filter design problem into a modelmatching problem. In the following theorem, a sufficient condition is provided for the existence of an admissible $H_{\infty}$ FD filter with the deficient transition probabilities (4).

Theorem 7. Consider system (1) with deficient transition information; for given $\xi>0$, determine the matrices $A_{F(g)}=$ $Q_{g(2)}^{-1} \bar{A}_{F(g)}, B_{F(g)}=Q_{g(2)}^{-1} \bar{B}_{F(g)}, C_{F(g)}=C_{f(g)}$, and $D_{F(g)}=$ $D_{f(g)}$; then the FD filter (6) is found so that the augmented error system (7) is randomly stable with an $H_{\infty}$ performance index $\xi$; if there exist positive-definite symmetric matrices $Q_{g}=$ $\left[\begin{array}{cc}Q_{g(1)} & W Q_{g(2)} \\ * & Q_{g(2)}\end{array}\right] \in R^{\left(n_{x}+n_{r}\right) \times\left(n_{x}+n_{r}\right)}, W:=\left[\begin{array}{ll}I_{n_{r}} & 0_{n_{r} \times\left(n_{x}-n_{r}\right)}\end{array}\right]^{T}$ and $M_{i}>0, \bar{A}_{F(g)}, \bar{B}_{F(g)}, C_{F(g)}, D_{F(g)}, \forall g \in S$, satisfy the following LMIs:

$$
\psi_{g j}^{(v)}=\left[\begin{array}{cccccc}
-I & \Theta_{1} & C_{F(g)} & 0 & \Theta_{2} & \Theta_{3} \\
* & \Theta_{4} & \Theta_{5} & \Theta_{6} & \Theta_{7} & \Theta_{8} \\
* & * & \Theta_{9} & \Theta_{10} & \Theta_{11} & \Theta_{12} \\
* & * & * & -\xi^{2} I & 0 & 0 \\
* & * & * & * & -\xi^{2} I & 0 \\
* & * & * & * & * & -\xi^{2} I
\end{array}\right]<0,
$$

$$
\begin{aligned}
& \tilde{\eta}_{g j}^{(v)}= \begin{cases}\eta_{k}^{(g)}+\eta_{u c}^{(g v)}-\left(\lambda_{k}^{(g)}+\lambda_{u c}^{(g v)}\right) \eta_{j}, & j \in S_{u k}^{(g)}, \text { if } g \in S_{k}^{(g)} \cup S_{u c}^{(g)}, \\
\eta_{k}^{(g)}+\eta_{u c}^{(g v)}+\lambda_{l}^{(g)} \eta_{g}-\left(\lambda_{l}^{(g)}+\lambda_{k}^{(g)}+\lambda_{u c}^{(g v)}\right) \eta_{j}, & j \in S_{u k}^{(g)}, \text { if } g \in S_{u k}^{(g)},\end{cases} \\
& \eta_{k}^{(g)}=\sum_{j \in S_{k}^{(g)}} \lambda_{g j} Q_{j},
\end{aligned}
$$

where 


$$
\begin{aligned}
\eta_{u c}^{(g v)} & =\sum_{j \in S_{u c}^{(g)}} \tilde{\lambda}_{g j}^{(v)} Q_{j}, \\
\lambda_{k}^{(g)} & =\sum_{j \in S_{k}^{(g)}} \lambda_{g j}, \\
\lambda_{u c}^{(g v)} & =\sum_{j \in S_{u c}^{(g)}} \tilde{\lambda}_{g j}^{(v)}, \\
\Theta_{1} & =D_{F(g)} C_{(g)}, \\
\Theta_{2} & =D_{F(g)} D_{(g)}, \\
\Theta_{3} & =D_{F(g)} H_{(g)}-I, \\
\Theta_{4} & =A_{(g)}^{T} Q_{g(1)}+Q_{g(1)} A_{(g)}+W \bar{B}_{F(g)} C_{(g)}+C_{(g)}^{T} \bar{B}_{F(g)}^{T} W^{T}+\widetilde{\eta}_{g j}^{(v)}, \\
\Theta_{5} & =A_{(g)}^{T} W Q_{g(2)}+W \bar{A}_{F(g)}+C_{(g)}^{T} \bar{B}_{F(g)}^{T}+\widetilde{\eta}_{g j}^{(v)}, \\
\Theta_{6} & =Q_{g(1)} B_{(g)}, \\
\Theta_{7} & =Q_{g(1)} E_{(g)}+W \bar{B}_{F(g)} D_{(g)}, \\
\Theta_{8} & =Q_{g(1)} F_{(g)}+W \bar{B}_{F(g)} H_{(g)}, \\
\Theta_{9} & =\bar{A}_{F(g)}^{T}+\bar{A}_{F(g)}+\widetilde{\eta}_{g j}^{(v)}, \\
\Theta_{10} & =Q_{g(2)}^{T} W^{T} B_{(g)}, \\
\Theta_{11} & =Q_{g(2)}^{T} W^{T} E_{(g)}+\bar{B}_{F(g)} D_{(g)}, \\
\Theta_{12} & =Q_{g(2)}^{T} W^{T} F_{(g)}+\bar{B}_{F(g)} H_{(g)} .
\end{aligned}
$$

Proof. For $H_{\infty}$ FD filter design purpose, we choose the slack matrix $Q_{g}$ as

$$
Q_{g}:=\left[\begin{array}{cc}
Q_{g(1)} & W Q_{(2)} \\
* & Q_{(3)}
\end{array}\right], \quad g \in S,
$$

where

$$
\begin{aligned}
W & :=\left[\begin{array}{ll}
I_{n_{r}} & O_{n_{r} \times\left(n_{x}-n_{r}\right)}
\end{array}\right]^{T}, \\
Q_{g(1)} & \in R^{n_{x} \times n_{x}}, \\
Q_{(2)} & \in R^{n_{r} \times n_{r}}, \\
Q_{(3)} & \in R^{n_{r} \times n_{r}} .
\end{aligned}
$$

Then, according to formula (24), performing the following congruent transformation, by $\left[\begin{array}{lll}I & 0 \\ * & \mathrm{Q}_{(2)} \mathrm{Q}_{(3)}^{-1}\end{array}\right]$, yields

$$
\begin{aligned}
& {\left[\begin{array}{lc}
I & 0 \\
0 & Q_{(2)} Q_{(3)}^{-1}
\end{array}\right]\left[\begin{array}{cc}
Q_{g(1)} & W Q_{(2)} \\
* & Q_{(3)}
\end{array}\right]\left[\begin{array}{cc}
I & 0 \\
0 & Q_{(2)} Q_{(3)}^{-1}
\end{array}\right]^{T}} \\
& \quad=\left[\begin{array}{cc}
Q_{g(1)} & W Q_{(2)} Q_{(3)}^{-1} Q_{(2)}^{T} \\
* & Q_{(2)} Q_{(3)}^{-1} Q_{(2)}^{T}
\end{array}\right]:=\left[\begin{array}{cc}
Q_{g(1)} & W Q_{g(2)} \\
* & Q_{g(2)}
\end{array}\right] .
\end{aligned}
$$

Thus, matrix $Q_{g}$ in (32) can been directly specified the following general form:

$$
Q_{g}=\left[\begin{array}{cc}
Q_{g(1)} & W Q_{g(2)} \\
* & Q_{g(2)}
\end{array}\right], \quad g \in S .
$$

It is noted that in this way the matrix variables $Q_{g(2)}$ are set as Markovian and can be absorbed directly by the gain variables $A_{F g}$ and $B_{F g}$ by introducing

$$
\begin{aligned}
& \bar{A}_{F g}=Q_{g(2)} A_{F g}, \\
& \bar{B}_{F g}=Q_{g(2)} B_{F g}, \\
& g \in S .
\end{aligned}
$$

Then we replace matrices $Q_{g}$ given by (33) into (11), together with the admissible filter parameter matrices defined in (8). Finally we can get (28) exactly. This completes the proof.

Remark 8. Up until now, it has been shown that the main results presented in Theorem 7 not only provide performance index $\xi^{*}$, but also give a numerically efficient and reliable approach to determine the corresponding gains of an admissible FD filter in (6) by using Matlab software. In order to 
acquire a receivable $H_{\infty}$ FD filter with $\xi$ made as small as possible in (9), it is necessary to calculate inequality (28) in Theorem 7 iteratively. Also, it can be derived from (28) that the design reduced-order FD filter and the corresponding error between residual and fault should be different on the basis of the different degree of deficient statistics of mode transitions. The main goal is to make the error as small as possible. To illustrate the feasibility and effectiveness of the proposed FD scheme, a numerical example will be given in the next section.

\section{Illustrative Examples}

For simplicity, we only consider two addressed FD examples for the continuous-time MJLSs with deficient transition information to demonstrate the effectiveness and practicability of the proposed approach.

Example 1. Consider (1) with four operation modes and the following matrices:

$$
\begin{aligned}
& A_{1}=\left[\begin{array}{cccc}
0 & 1 & 0 & 0 \\
6.34 & 23.66 & 32.83 & 35.51 \\
0 & 0 & 0 & 2 \\
-12.46 & -48.71 & -54.25 & -67.50
\end{array}\right] \text {, } \\
& A_{2}=\left[\begin{array}{cccc}
0 & 2 & 0 & 0 \\
5.34 & 22.66 & 31.83 & 34.51 \\
0 & 0 & 0 & 1 \\
-11.46 & -47.71 & -53.25 & -66.50
\end{array}\right] \text {, } \\
& A_{3}=\left[\begin{array}{cccc}
0 & 1 & 0 & 0 \\
4.34 & 21.66 & 29.83 & 31.51 \\
0 & 0 & 0 & 3 \\
-9.46 & -44.71 & -51.25 & -63.50
\end{array}\right] \text {, } \\
& A_{4}=\left[\begin{array}{cccc}
0 & 3 & 0 & 0 \\
8.34 & 28.66 & 35.83 & 37.51 \\
0 & 0 & 0 & 5 \\
-17.46 & -53.71 & -57.25 & -73.50
\end{array}\right] \text {, } \\
& B_{1}=[0 ; 0.2361 ; 0 ;-0.4758] \text {, } \\
& B_{2}=[0 ; 0.1209 ; 0 ;-0.3621] \text {, } \\
& B_{3}=[0 ; 0.2341 ; 0 ;-0.7653] \text {, } \\
& B_{4}=[0 ; 0.7451 ; 0 ;-0.5832] \text {, } \\
& C_{1}=\left[\begin{array}{llll}
1 & 0 & 0 & 0
\end{array}\right] \text {, } \\
& C_{2}=\left[\begin{array}{llll}
1 & 0 & 3 & 0
\end{array}\right] \text {, } \\
& C_{3}=\left[\begin{array}{llll}
1 & 2 & 0 & 0
\end{array}\right] \text {, } \\
& C_{4}=\left[\begin{array}{llll}
0 & 1 & 0 & 0
\end{array}\right] \text {, } \\
& D_{1}=D_{2}=D_{3}=D_{4}=0.5 \text {, } \\
& E_{1}=E_{2}=E_{3}=E_{4}=\left[\begin{array}{llll}
0 & 0.5 & 0 & -0.5
\end{array}\right] \text {, } \\
& F_{1}=F_{2}=F_{3}=F_{4}=\left[\begin{array}{llll}
0 & -1 & 0 & 0.3
\end{array}\right], \\
& H_{1}=H_{2}=H_{3}=H_{4}=0.1 \text {. }
\end{aligned}
$$

In order to make the simulation simplification, we consider the exogenous disturbance input $\omega(t)=0.15 \cos (t)$ for $0 \leq t \leq 200$. The fault signal $f(t)$ is

$$
f(t)= \begin{cases}0.8, & 60 \leq t \leq 120 \\ 0, & \text { others }\end{cases}
$$

Now, four cases for different transition rate matrix (TRM) are shown as follows.

\section{Four Different TRMs}

Case 1 (completely known TRM).

$$
\left[\begin{array}{cccc}
-0.8 & 0.3 & 0.1 & 0.4 \\
0.7 & -1.2 & 0.3 & 0.2 \\
0.1 & 0.5 & -1.3 & 0.7 \\
0.2 & 0.2 & 0.1 & -0.5
\end{array}\right] \text {. }
$$

Case 2 (polytopic uncertain TRM).

$$
\left[\begin{array}{cccc}
-0.8 & 0.3 & \hat{\lambda}_{13} & \hat{\lambda}_{14} \\
\hat{\lambda}_{21} & \hat{\lambda}_{22} & \hat{\lambda}_{23} & \hat{\lambda}_{24} \\
\tilde{\lambda}_{31} & \hat{\lambda}_{32} & \tilde{\lambda}_{33} & \hat{\lambda}_{34} \\
\hat{\lambda}_{41} & \hat{\lambda}_{42} & 0.1 & -0.5
\end{array}\right]
$$

Case 3 (partly known TRM).

$$
\left[\begin{array}{cccc}
-0.8 & 0.3 & \hat{\lambda}_{13} & \hat{\lambda}_{14} \\
\hat{\lambda}_{21} & \hat{\lambda}_{22} & 0.3 & \hat{\lambda}_{24} \\
\hat{\lambda}_{31} & \hat{\lambda}_{32} & \hat{\lambda}_{33} & \hat{\lambda}_{34} \\
\hat{\lambda}_{41} & \hat{\lambda}_{42} & 0.1 & -0.5
\end{array}\right] .
$$

Case 4 (completely unknown TRM).

$$
\left[\begin{array}{llll}
\hat{\lambda}_{11} & \hat{\lambda}_{12} & \hat{\lambda}_{13} & \hat{\lambda}_{14} \\
\hat{\lambda}_{21} & \hat{\lambda}_{22} & \hat{\lambda}_{23} & \hat{\lambda}_{24} \\
\hat{\lambda}_{31} & \hat{\lambda}_{32} & \hat{\lambda}_{33} & \hat{\lambda}_{34} \\
\hat{\lambda}_{41} & \hat{\lambda}_{42} & \hat{\lambda}_{43} & \hat{\lambda}_{44}
\end{array}\right] .
$$

And the simulation result of Markov chain $\varsigma(t)$ is given in Figure 1.

For Case 2, the TRM includes two vertices $\Delta_{\zeta}, \varsigma=1,2$, and their third row $\Delta_{\varsigma}^{2}, \varsigma=1,2$, is given by

$$
\begin{aligned}
& \Delta_{1}^{2}=\left[\begin{array}{llll}
0.6 & \hat{\lambda}_{32} & -1.1 & \hat{\lambda}_{34}
\end{array}\right], \\
& \Delta_{2}^{2}=\left[\begin{array}{llll}
0.42 & \hat{\lambda}_{32} & -0.93 & \hat{\lambda}_{34}
\end{array}\right] .
\end{aligned}
$$

Applying Theorem 7 through the Matlab LMI Toolbox, the gains of an admissible FD filter in the form of (6) for four different TRMs as shown above are acquired, respectively. 
TABLE 1: Computation results for four different reduced-order FD filters cases.

\begin{tabular}{lcc}
\hline Transition rate matrix & $J_{\min }$ & Time steps \\
\hline Completely known (Case 1) & 1.0002 & 61 \\
Polytopic uncertain (Case 2) & 1.0004 & 62 \\
Partly known (Case 3) & 1.0007 & 63 \\
Completely unknown (Case 4) & 1.0010 & 64 \\
\hline
\end{tabular}

TABLE 2: Computation results for four different full-order FD filters cases.

\begin{tabular}{lcc}
\hline Transition rate matrix & $J_{\min }$ & Time steps \\
\hline Completely known (Case 1) & 1.0001 & 62 \\
Polytopic uncertain (Case 2) & 1.0002 & 63 \\
Partly known (Case 3) & 1.0005 & 64 \\
Completely unknown (Case 4) & 1.0008 & 65 \\
\hline
\end{tabular}

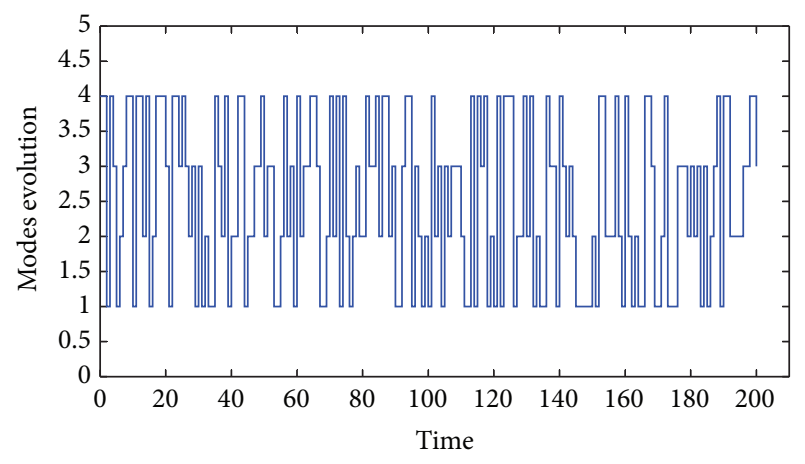

Figure 1: Modes evolution in Example 1.

Obviously, it is seen from Figure 2, which presents the generated residual signals $r(t)$, that the more the transition rate information we have known is, the smaller the generated residual $r(t)$ will become; for example, the generated residual value in Case 2 is the smaller than the residual value in Case 3. The simulation of polytopic uncertain TRs has better result than that of partly known TRs or completely unknown TRs.

In the following, Figure 3 displays the evolution of $J\left(r_{e}\right)=$ $\sqrt{\int_{k_{0}}^{k_{0}+e} r^{T}(t) r(t) d t}$ for both faulty case and fault-free case, respectively. It can be concluded from Figure 3 that when the fault occurs, the residual and the residual evaluation function have obvious change and the $H_{\infty}$ performance indices for the error augmented system (7) in Case 2 are better than those in Cases 3 and 4 .

According to the path in Figure 1 and the residual threshold $J_{\text {th }}=\sup _{d \in l_{2}, f=0} E\left[\sqrt{\int_{k_{0}}^{k_{0}+L} r^{T}(t) r(t) d t}\right]$, for the four different TRM cases, the optimal $H_{\infty}$ performance indices and the corresponding time steps for the FD are obtained in Tables 1 and 2. The filter gain is set to 0.1. From the computation results, it can be also shown that the FD capability in Case 2 is stronger than that in Cases 3 and 4 .

Remark 9. From the comparison results of the same-order FD filters, it is clear to see that the fault detection results in polytopic uncertain TRs are less conservative than those in incompletely known and completely unknown TRs. The more the polytopic uncertain knowledge in the TRM is, the faster the sensitivity to faults will be taken and the better the fault detection performance the filter can attain is. The time steps to detect the fault have been shortened. Finally, comparing Tables 1 and 2, we can find that FD speed with the reduced-order filter is faster than that with full-order filter in the same case. Thus, it declares the effectiveness of the designed FD reduced-order filter for MJLSs with deficient transition information.

Example 2. Consider a vertical take-off and landing (VTOL) helicopter system (see [2]). The system dynamics can be modeled as

$$
\begin{aligned}
& \dot{x}(t)=A(\varsigma(t)) x(t)+B(\varsigma(t)) u(t)+E(\varsigma(t)) \omega(t), \\
& y(t)=C(\varsigma(t)) x(t)+D(\varsigma(t)) \omega(t) .
\end{aligned}
$$

The behavior of $\varsigma(t)$ is characterized as a Markov chain with three different modes, corresponding to airspeeds of 135 , 60 , and 170 knots, respectively.

$$
A_{1}(\varsigma(t))=\left[\begin{array}{cccc}
-0.0366 & 0.0271 & 0.0188 & -0.4555 \\
0.0482 & -1.01 & 0.0024 & -4.0208 \\
0.1002 & 0.3681 & -0.707 & 1.4200 \\
0 & 0 & 1 & 0
\end{array}\right] \text {, }
$$$$
A_{2}(\varsigma(t))=\left[\begin{array}{cccc}
-0.0366 & 0.0271 & 0.0188 & -0.4555 \\
0.0482 & -1.01 & 0.0024 & -4.0208 \\
0.1002 & 0.0664 & -0.707 & 0.1198 \\
0 & 0 & 1 & 0
\end{array}\right] \text {, }
$$$$
A_{3}(\varsigma(t))=\left[\begin{array}{cccc}
-0.0366 & 0.0271 & 0.0188 & -0.4555 \\
0.0482 & -1.01 & 0.0024 & -4.0208 \\
0.1002 & 0.5047 & -0.707 & 2.5460 \\
0 & 0 & 1 & 0
\end{array}\right] \text {, }
$$

$$
\begin{aligned}
& B_{1}(\varsigma(t))=[0.4422 ; 3.5446 ;-5.5200 ; 0] \text {, } \\
& B_{2}(\varsigma(t))=[0.4422 ; 0.9775 ;-5.5200 ; 0] \text {, } \\
& B_{3}(\varsigma(t))=[0.4422 ; 5.1120 ;-5.5200 ; 0] \text {, } \\
& C_{1}(\varsigma(t))=C_{2}(\varsigma(t))=C_{3}(\varsigma(t))=\left[\begin{array}{llll}
0 & 0.5 & 0 & 0.6
\end{array}\right] \text {, } \\
& D_{1}(\varsigma(t))=D_{2}(\varsigma(t))=D_{3}(\varsigma(t))=0.3 \text {, } \\
& E_{1}(\varsigma(t))=E_{2}(\varsigma(t))=E_{3}(\varsigma(t)) \\
& =[0.1761 ;-7.5922 ; 4.4900 ; 0] \text {. }
\end{aligned}
$$



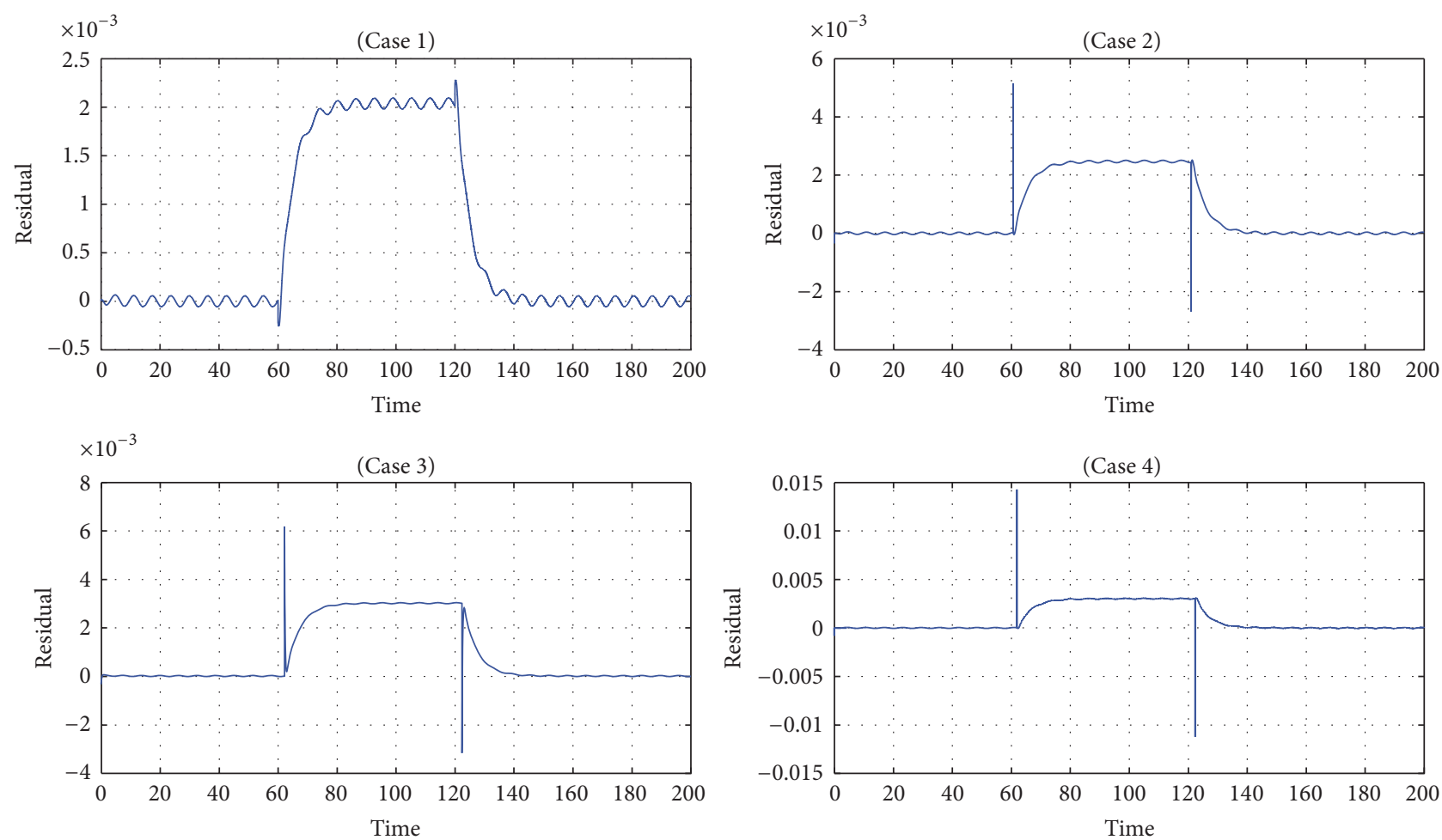

Figure 2: Generated residual in Example 1.
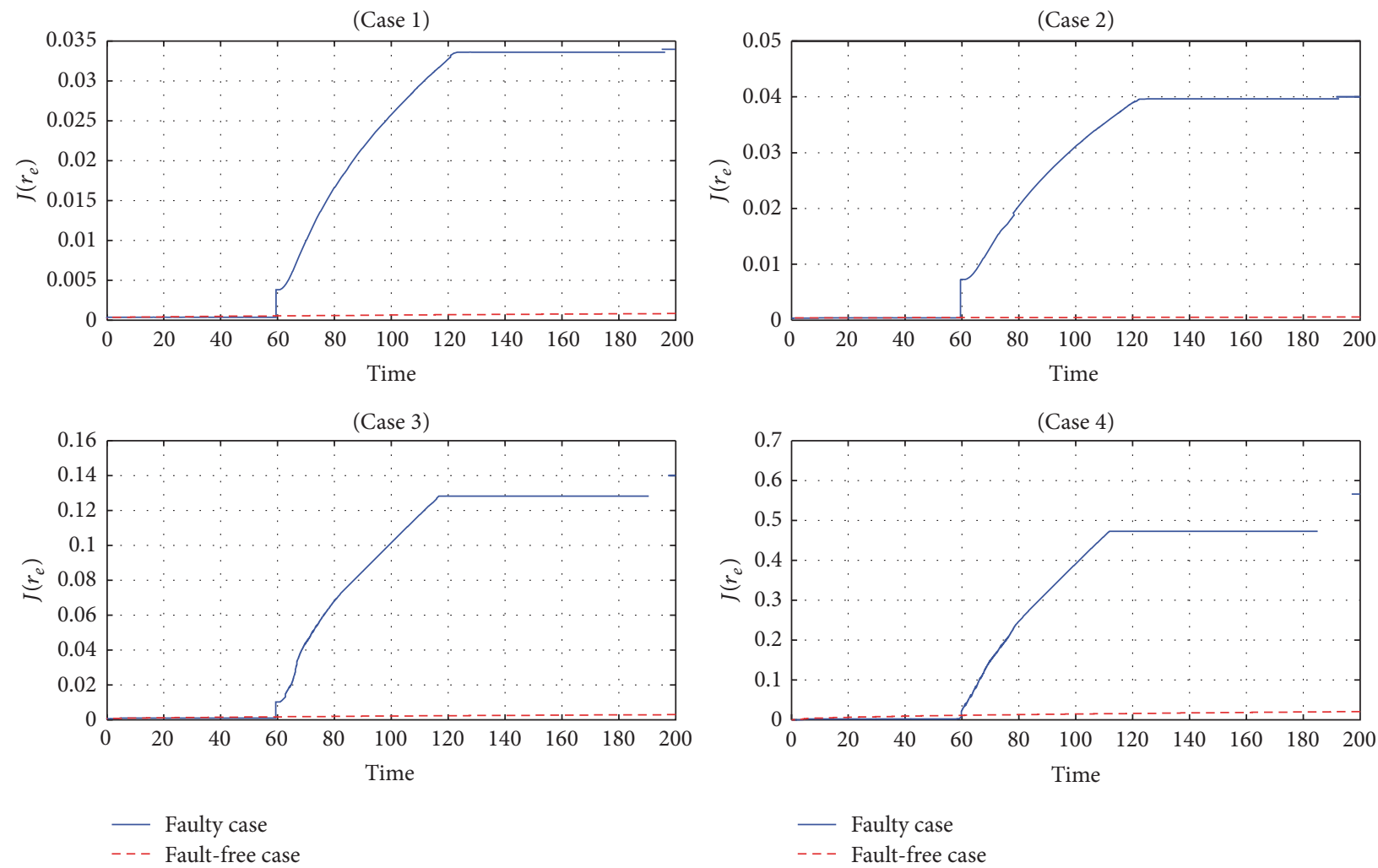

- Faulty case

- - - Fault-free case

FIgURE 3: Evolution of $J\left(r_{e}\right)$ in Example 1. 


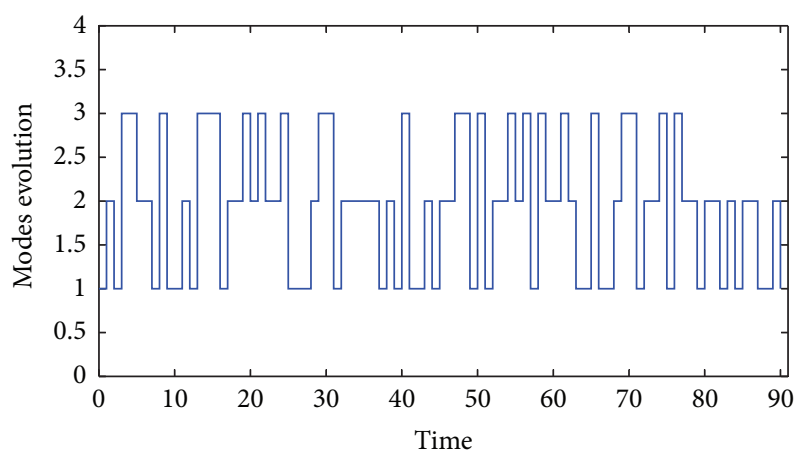

FIgure 4: Modes evolution in Example 2.

The system state is $x=\left[x_{1} ; x_{2} ; x_{3} ; x_{4}\right]$, where $x_{1}$ is the horizontal velocity, $x_{2}$ is the vertical velocity, $x_{3}$ is the pitch rate, and $x_{4}$ is the pitch angle. In order to make the simulation simplification, one type of the exogenous disturbance signal on the side-slip sensor is only considered in the VTOL helicopter example. we consider the system input $u(t)=$ $\sin (t)$ for $0 \leq t \leq 90$. The exogenous disturbance signal is $\omega(t)=\operatorname{sawtooth}(0.1 * t), 0 \leq t \leq 90$. Now, four different transition rate matrices (TRMs) are shown as follows.

\section{Four Different TRMs}

Case 1 (completely known TRM).

$$
\left[\begin{array}{ccc}
-0.9 & 0.3 & 0.6 \\
0.7 & -1.2 & 0.5 \\
0.8 & 0.5 & -1.3
\end{array}\right] \text {. }
$$

Case 2 (polytopic uncertain TRM).

$$
\left[\begin{array}{ccc}
-0.9 & 0.3 & 0.6 \\
\tilde{\lambda}_{21} & \tilde{\lambda}_{22} & \tilde{\lambda}_{23} \\
\hat{\lambda}_{31} & \widehat{\lambda}_{32} & -1.3
\end{array}\right] .
$$

Case 3 (partly known TRM).

$$
\left[\begin{array}{ccc}
-0.9 & 0.3 & 0.6 \\
\widehat{\lambda}_{21} & \widehat{\lambda}_{22} & \widehat{\lambda}_{23} \\
\widehat{\lambda}_{31} & \widehat{\lambda}_{32} & -1.3
\end{array}\right] .
$$

Case 4 (completely unknown TRM).

$$
\left[\begin{array}{lll}
\hat{\lambda}_{11} & \hat{\lambda}_{12} & \hat{\lambda}_{13} \\
\hat{\lambda}_{21} & \hat{\lambda}_{22} & \hat{\lambda}_{23} \\
\hat{\lambda}_{31} & \hat{\lambda}_{32} & \widehat{\lambda}_{33}
\end{array}\right] .
$$

And the simulation result of three switching cases for Markov chain $\varsigma(t)$ is given in Figure 4 .
For Case 2, the TRM includes two vertices $\Delta_{\varsigma}, \varsigma=1,2$, and their second row $\Delta_{\varsigma}^{2}, \varsigma=1,2$, is given by

$$
\begin{aligned}
& \Delta_{1}^{2}=\left[\begin{array}{lll}
0.6 & -1.1 & 0.5
\end{array}\right], \\
& \Delta_{2}^{2}=\left[\begin{array}{lll}
0.4 & -1.7 & 1.3
\end{array}\right] .
\end{aligned}
$$

Applying Theorem 7 through the Matlab LMI Toolbox, the gains of an admissible FD filter in the form of (6) for four different TRMs as shown above are acquired, respectively. This means that the fault detection filter of VTOL helicopter system can be designed. By using this filter, the generated residual $r(t)$ and $J_{r(t)}$ values can be calculated.

Obviously, it is seen from Figure 5 that the more the transition rate information we have known, the closer the estimates of the disturbance signal to the real values of the interference signal. The detected residual signal $r(t)$ in Case 2 is more accurate than that in Case 3. The fault detection time in Case 2 is shorter than those of in Cases 3 and 4.

Figure 6 displays the evolution of $J_{r(t)}$ for both disturbance case and disturbance-free case, respectively. Because the disturbance signal is not equal to the fault signal, when the fault occurs, the side-slip angle sensor should be switched to the other redundant sensor. For example, when $J_{\text {th }}$ is 0.5 , the disturbance case can be accurately judged in Cases 1 and 2, but it is wrong to judge as the failure condition in Cases 3 and 4. The performance of fault detection filtering for the error augmented system (7) in Case 2 is better than that in Case 3 or Case 4, because it can reduce the rate of false positives.

\section{Conclusion}

In this paper, a fault detection approach is proposed for continuous-time MJLSs with deficient transition information. The main contribution of our study is the introduction of Markov jump system with deficient transition information in fault detection reduced-order filter design. Special emphasis is that the abovementioned method is used in the fault detection process of continuous-time MJLSs for the first time. The underlying systems are more general, because the deficient transition descriptions include completely known, polytopic uncertain, partly unknown, and completely unknown transition rates. Based on the linear matrix inequality approach and the linear convex optimization, a sufficient condition of FD reduced-order filter for continuous-time MJLS with deficient transition information is obtained, such that the augmented error system is randomly stable. Then, the changes of the fault signal are approximately equal to the changes of the residual signal. Finally, the two simulation examples have been given to illustrate the effectiveness of the proposed design approach, which can improve the sensitivity of fault detection and reduce the fault detection rate of false positives. Due to the fact that time-varying delay is found in the process of fault detection, some interesting topics for future works include fault detection and fault-tolerant control methods for time-varying delay and uncertain process in the different Markov systems. 

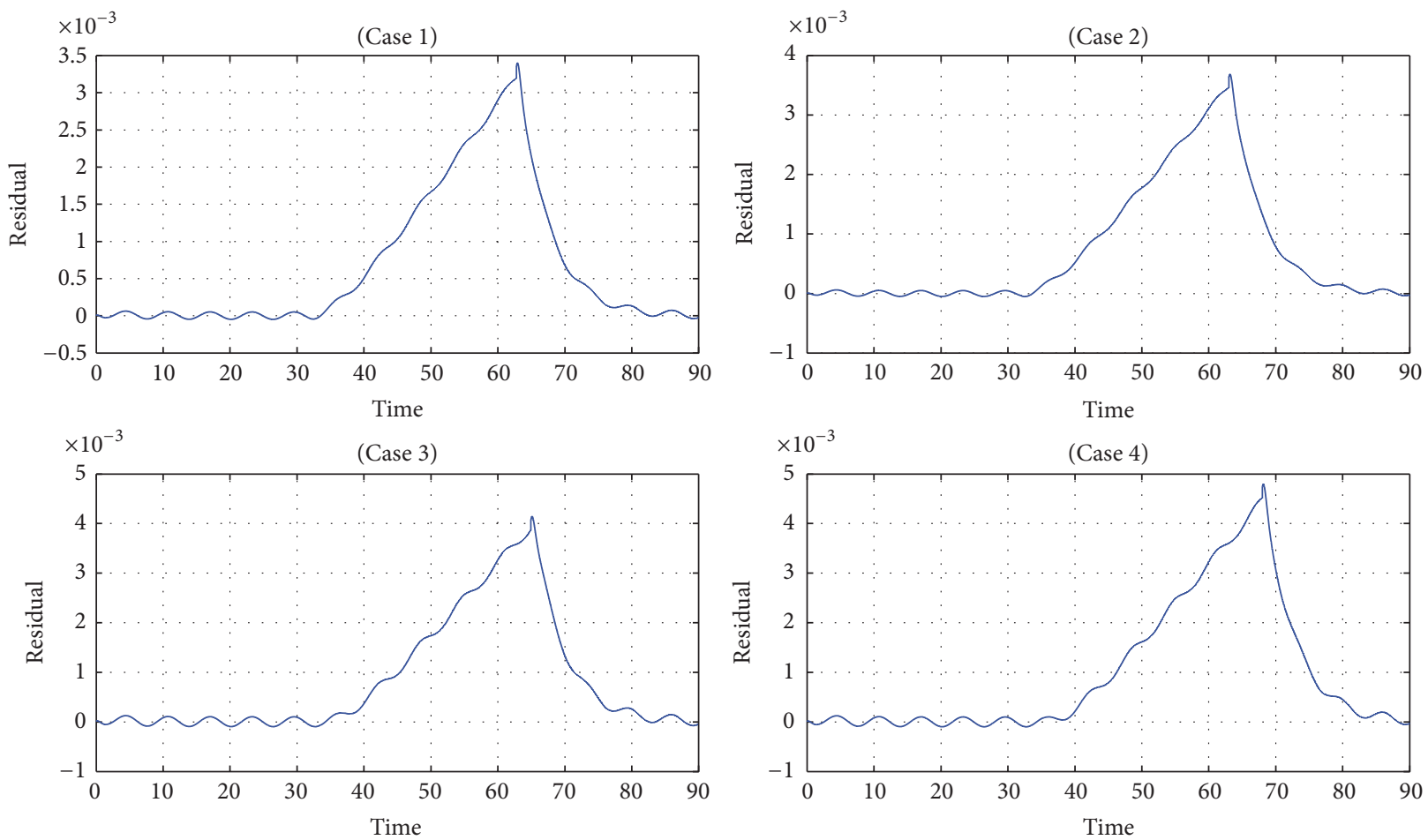

FIGURE 5: Generated residual in Example 2.
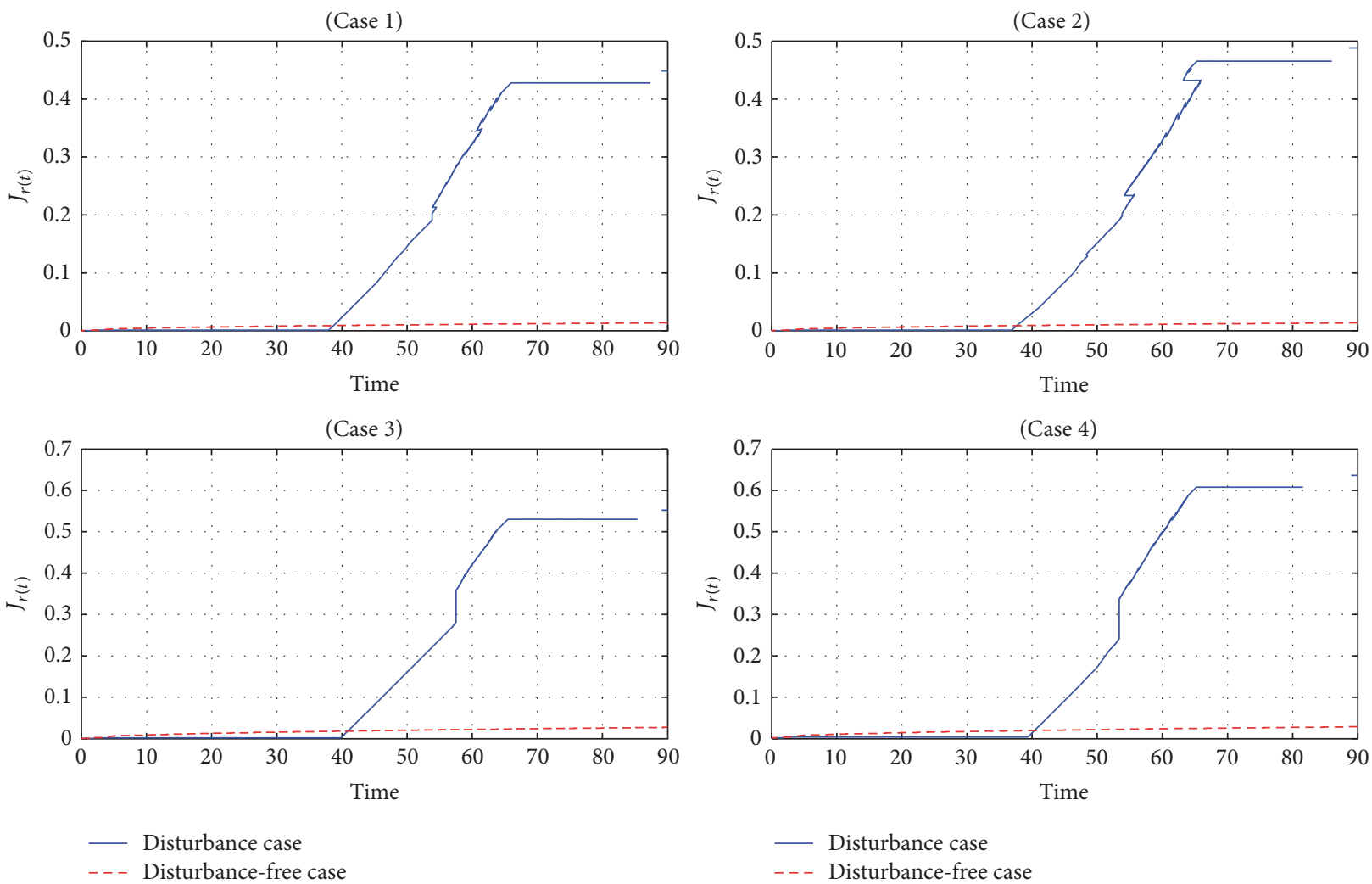

Disturbance-free case

_. - Disturbance-free case

FIgURE 6: Evolution of $J_{r(t)}$ in Example 2. 


\section{Competing Interests}

The authors declare that they have no competing interests.

\section{Acknowledgments}

This work was supported by the National Natural Science Foundation of China (Grant no. 61503091) and the China Postdoctoral Science Foundation (2016T90270, 2015M570282) and the Postdoctoral Science Foundation of Heilongjiang Province (LBH-Z14056).

\section{References}

[1] P. Bolzern, P. Colaneri, and G. De Nicolao, "Markov jump linear systems with switching transition rates: mean square stability with dwell-time," Automatica, vol. 46, no. 6, pp. 1081-1088, 2010.

[2] J. Qiu, Y. Wei, and H. R. Karimi, "New approach to delaydependent $H_{\infty}$ control for continuous-time Markovian jump systems with time-varying delay and deficient transition descriptions," Journal of the Franklin Institute, vol. 352, no. 1, pp. 189-215, 2015.

[3] Y. Wei, X. Peng, J. Qiu, and S. Jia, " $H_{\infty}$ filtering for twodimensional continuous-time Markovian jump systems with deficient transition descriptions," Neurocomputing, vol. 167, pp. 406-417, 2015.

[4] Y. Wei, M. Wang, and J. Qiu, "New approach to delay-dependent $H_{\infty}$ filtering for discrete-time Markovian jump systems with time-varying delay and incomplete transition descriptions," IET Control Theory \& Applications, vol. 7, no. 5, pp. 684-696, 2013.

[5] L. Zhang, E.-K. Boukas, L. Baron, and H. R. Karimi, "Fault detection for discrete-time Markov jump linear systems with partially known transition probabilities," International Journal of Control, vol. 83, no. 8, pp. 1564-1572, 2010.

[6] M. Zhong, H. Ye, and P. Shi, "Fault detection for Markovian jump systems," IEE Proceedings Control Theory and Applications, vol. 152, no. 4, pp. 397-402, 2005.

[7] Y. Wei, J. Qiu, and H. R. Karimi, "Reliable output feedback control of discretetime fuzzy affine systems with actuator faults," IEEE Transactions on Circuits and Systems-I: Regular Papers, In press.

[8] Y. Wei, J. Qiu, and S. Fu, "Mode-dependent nonrational output feedback control for continuous-time semi-Markovian jump systems with time-varying delay," Nonlinear Analysis. Hybrid Systems, vol. 16, pp. 52-71, 2015.

[9] J. Qiu, S. X. Ding, H. Gao, and S. Yin, "Fuzzy-model-based reliable static output feedback control of nonlinear hyperbolic pde systems," IEEE Transactions on Fuzzy Systems, vol. 24, no. 2, pp. 388-400, 2016.

[10] Y. Wei, J. Qiu, H. R. Karimi, and M. Wang, "New results on $H_{\infty}$ dynamic output feedback control for Markovian jump systems with time-varying delay and defective mode information," Optimal Control Applications and Methods, vol. 35, no. 6, pp. 656-675, 2014.

[11] Y. Wei, J. Qiu, H. Lam, and L. Wu, "Approaches to T-S fuzzy-affine-model-based reliable output feedback control for nonlinear ito stochastic systems," IEEE Transactions on Fuzzy Systems, 2016.

[12] X.-H. Chang, "Robust nonfragile $H_{\infty}$ filtering of fuzzy systems with linear fractional parametric uncertainties," IEEE Transactions on Fuzzy Systems, vol. 20, no. 6, pp. 1001-1011, 2012.
[13] A. Ahadi, N. Ghadimi, and D. Mirabbasi, "An analytical methodology for assessment of smart monitoring impact on future electric power distribution system reliability," Complexity, vol. 21, no. 1, pp. 99-113, 2015.

[14] Y. Lu, W. Ren, S. Yi, and Y. Zuo, "Stability analysis for discrete delayed Markovian jumping neural networks with partly unknown transition probabilities," Neurocomputing, vol. 74, no. 18, pp. 3768-3772, 2011.

[15] R. Sakthivel, S. Selvi, K. Mathiyalagan, and A. Arunkumar, "Robust reliable sampled-data $H_{\infty}$ control for uncertain stochastic systems with random delay," Complexity, vol. 21, no. 1, pp. 42-58, 2015.

[16] O. L. Costa and G. R. Benites, "Linear minimum mean square filter for discrete-time linear systems with Markov jumps and multiplicative noises," Automatica, vol. 47, no. 3, pp. 466-476, 2011.

[17] P. Bolzern, P. Colaneri, and G. D. Nicolao, "Markov jump linear systems with switching transition rates: mean square stability with dwell-time," Automatica, vol. 46, no. 6, pp. 1081-1088, 2010.

[18] I. Hwang, S. Kim, Y. Kim, and C. E. Seah, "A survey of fault detection, isolation, and reconfiguration methods," IEEE Transactions on Control Systems Technology, vol. 18, no. 3, pp. 636-653, 2010.

[19] G. Gagliardi, A. Casavola, and D. Famularo, "A fault detection filter design method for Markov jump linear parameter varying systems," in Proceedings of the 7th IFAC International Symposium on Fault Detection, Supervision and Safety of Technical Systems (SAFEPROCESS '09), pp. 426-431, Barcelona, Spain, July 2009.

[20] I. Shames, A. M. H. Teixeira, H. Sandberg, and K. H. Johansson, "Distributed fault detection for interconnected second-order systems," Automatica, vol. 47, no. 12, pp. 2757-2764, 2011.

[21] M. Faraji-Niri, M. R. Jahed-Motlagh, and M. BarkhordariYazdi, "Stabilization of active fault-tolerant control systems by uncertain nonhomogeneous markovian jump models," Complexity, vol. 21, no. 1, pp. 318-329, 2016.

[22] Y. Wei, J. Qiu, H. R. Karimi, and M. Wang, "Model approximation for two-dimensional Markovian jump systems with statedelays and imperfect mode information," Multidimensional Systems and Signal Processing, vol. 26, no. 3, pp. 575-597, 2015.

[23] Y. C. Ding, H. Zhu, S. M. Zhong, and Y. P. Zhang, "Fault detection filter for Markov jump system," Advanced Materials Research, vol. 503, no. 4, pp. 1488-1492, 2012.

[24] Z. Mao, B. Jiang, and P. Shi, " $H_{\infty}$ fault detection filter design for networked control systems modelled by discrete Markovian jump systems," IET Control Theory \& Applications, vol. 1, no. 5, pp. 1336-1343, 2007.

[25] L. Rong, X. Peng, and B. Zhang, "Fault detection reducedorder filter design for discrete-time Markov jump system with deficient transition information," EURASIP Journal on Advances in Signal Processing, 2016.

[26] E. Frisk and L. Nielsen, "Robust residual generation for diagnosis including a reference model for residual behavior," Automatica, vol. 42, no. 3, pp. 437-445, 2006.

[27] R. Isermann, "Supervision, fault-detection and fault-diagnosis methods-an introduction," Control Engineering Practice, vol. 5, no. 5, pp. 639-652, 1997.

[28] P. M. Frank, "Fault diagnosis in dynamic systems using analytical and knowledge-based redundancy. A survey and some new results," Automatica, vol. 26, no. 3, pp. 459-474, 1990. 
[29] M. Zhong, S. X. Ding, and E. L. Ding, "Optimal fault detection for linear discrete time-varying systems," Automatica, vol. 46, no. 8, pp. 1395-1400, 2010.

[30] J. Gertler, "Fault detection and isolation using parity relations," Control Engineering Practice, vol. 5, no. 5, pp. 653-661, 1997.

[31] Y. Liu, S. M. Lee, O. M. Kwon, and J. H. Park, "Delay-dependent exponential stability criteria for neutral systems with interval time-varying delays and nonlinear perturbations," Journal of the Franklin Institute, vol. 350, no. 10, pp. 3313-3327, 2013.

[32] M. Chadli, A. Abdo, and S. X. Ding, " $H_{-} / H_{\infty}$ fault detection filter design for discrete-time Takagi-Sugeno fuzzy system," Automatica, vol. 49, no. 7, pp. 1996-2005, 2013.

[33] C. Keliris, M. M. Polycarpou, and T. Parisini, "A distributed fault detection filtering approach for a class of interconnected continuous-time nonlinear systems," IEEE Transactions on Automatic Control, vol. 58, no. 8, pp. 2032-2047, 2013.

[34] Y. Wei, J. Qiu, H. R. Karimi, and M. Wang, "Filtering design for two-dimensional Markovian jump systems with state-delays and deficient mode information," Information Sciences, vol. 269, pp. 316-331, 2014.

[35] Y. Wei, X. Peng, and J. Qiu, "Robust and non-fragile static output feedback control for continuous-time semi-Markovian jump systems," Transactions of the Institute of Measurement and Control, vol. 38, no. 9, pp. 1136-1150, 2016.

[36] Y. Wei, J. Qiu, P. Shi, and H.-K. Lam, "A new design of H-infinity piecewise filtering for discrete-time nonlinear time-varying delay systems via T-S fuzzy affine models," IEEE Transactions on Systems, Man, and Cybernetics: Systems, 2016.

[37] Z. Hameed, Y. S. Hong, Y. M. Cho, S. H. Ahn, and C. K. Song, "Condition monitoring and fault detection of wind turbines and related algorithms: a review," Renewable and Sustainable Energy Reviews, vol. 13, no. 1, pp. 1-39, 2009.

[38] Y. Wei, J. Qiu, and H. R. Karimi, "Quantized $H_{\infty}$ filtering for continuous-time Markovian jump systems with deficient mode information," Asian Journal of Control, vol. 17, no. 5, pp. 19141923, 2015.

[39] M. T. Raza, A. Q. Khan, G. Mustafa, and M. Abid, "Design of fault detection and isolation filter for switched control systems under asynchronous switching," IEEE Transactions on Control Systems Technology, vol. 24, no. 1, pp. 13-23, 2015. 


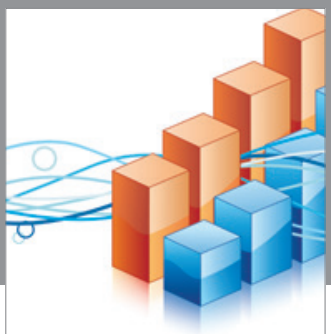

Advances in

Operations Research

vatem alat4

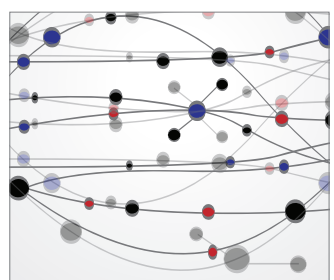

\section{The Scientific} World Journal
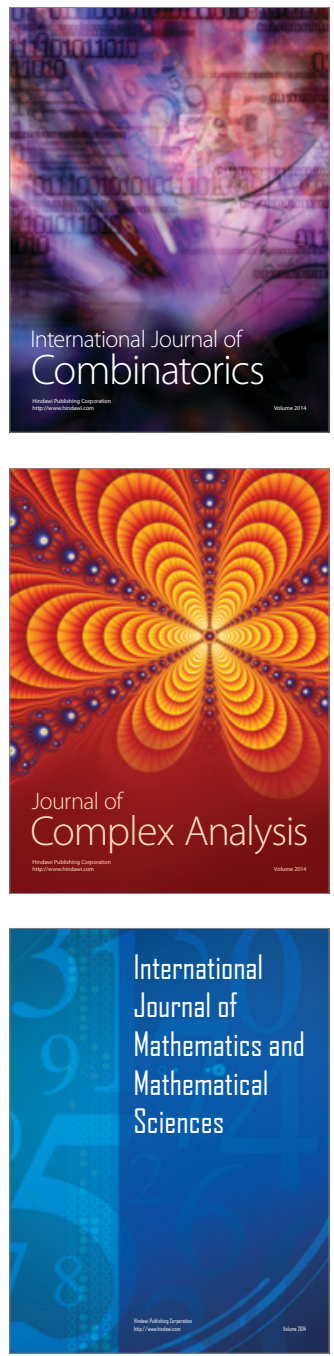
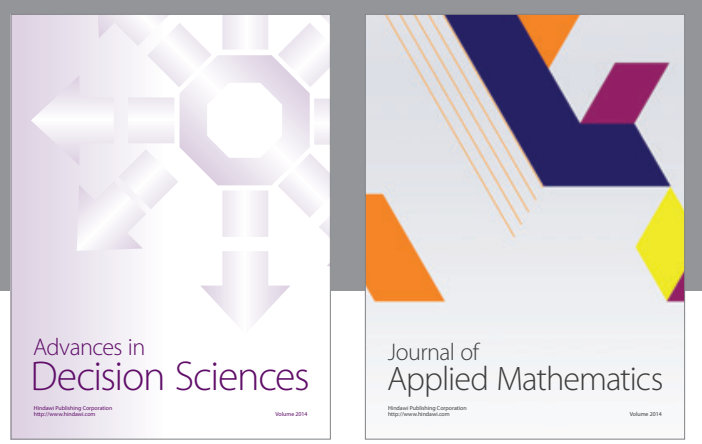

Algebra

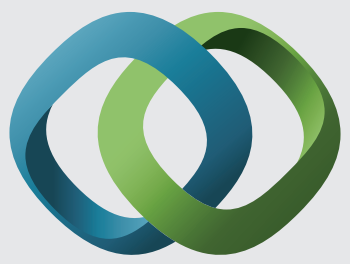

\section{Hindawi}

Submit your manuscripts at

https://www.hindawi.com
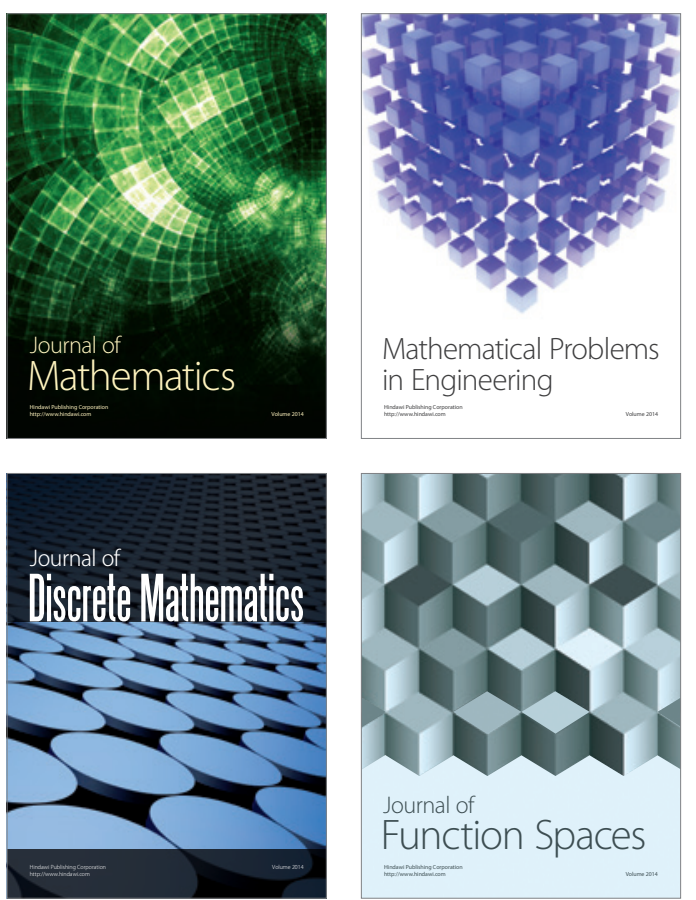

Mathematical Problems in Engineering
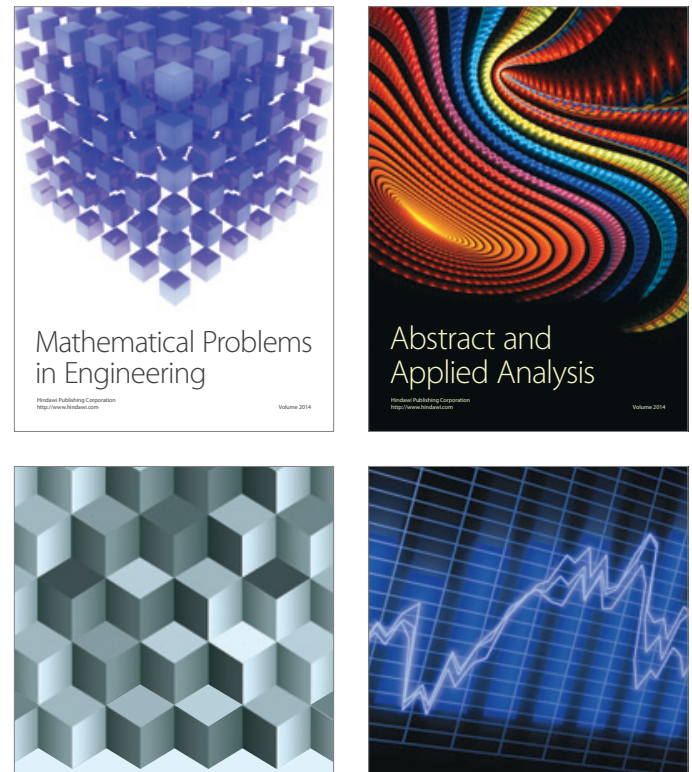

Journal of

Function Spaces

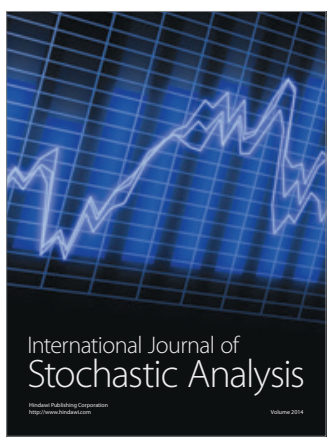

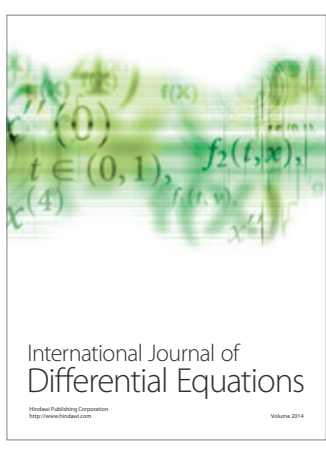
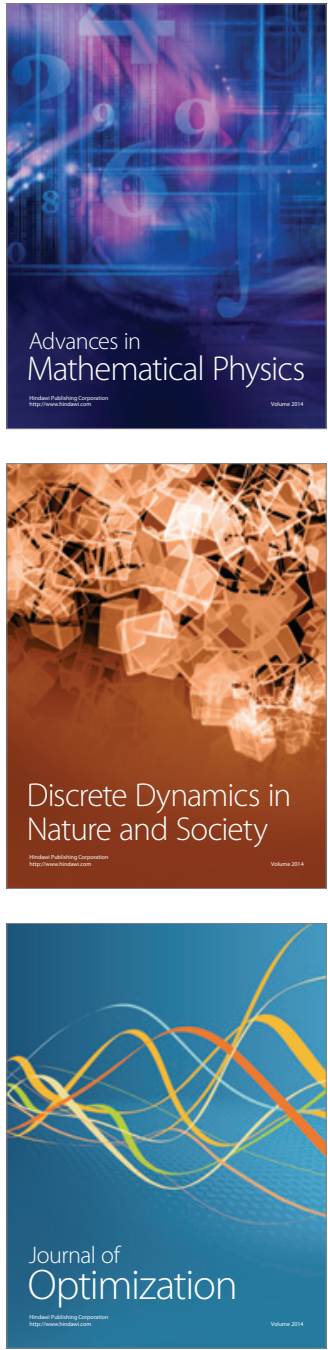